\title{
PERFIL EPIDEMIOLÓGICO DA COINFECÇÃO TUBERCULOSE/HIV NO PERIODO DE 2015 A 2019 NO BRASIL: UMA REVISAO DA LITERATURA
}

\author{
EPIDEMIOLOGICAL PROFILE OF TUBERCULOSIS / HIV \\ COINFECTION IN THE PERIOD FROM 2015 TO 2019 IN BRAZIL: A \\ LITERATURE REVIEW
}

\author{
Anne Caroline de Souza ${ }^{1}$ \\ Vanessa Rodrigues Fernandes ${ }^{2}$ \\ Ankilma do Nascimento Andrade Feitosa ${ }^{3}$ \\ Sheylla Nadjane Batista Lacerda \\ Tânia Maria Ribeiro Monteiro de Figueiredo \\ Talina Carla da Silva
}

RESUMO: OBJETIVO: analisar os aspectos epidemiológicos da coinfecção Tuberculose/HIV no Brasil. MÉTODOS: Trata-se de uma revisão integrativa da literatura, pautada a partir da seguinte questão norteadora: Qual o perfil epidemiológico da coinfecção tuberculose/hiv no periodo de 2015 a 2019 no Brasil? Como critérios de inclusão definiram-se: artigos publicados entre os anos de 2015 a 2019, em português e inglês, disponíveis na integra e gratuitos e que abordassem a temática de coinfecção TB/HIV. Para os critérios de exclusão foram utilizados: trabalhos não disponíveis, material de congressos, aula, tese, resumo ou conferencias; revisões da literatura, bem como artigos que não abordavam o tema proposto ou não estavam entre os anos definidos. A busca pelos artigos ocorreu pela Biblioteca Virtual de Saúde (BVS), que indexa bases de dados como Medical Literature Analysis and Retrieval Sistem (MEDLINE) e Literatura Latino-Americana e do Caribe em Ciências da Saúde (LILACS) através dos termos obtidos por meio de consulta aos DeCs: "HIV", "Tuberculose", "Coinfecção", "Brasil" no mês de fevereiro de 2020. RESULTADOS: Pela análise dos resultados encontrados nesta revisão, identificou-se um padrão de prevalência de estudos que apresentaram o perfil sociodemográfico, seja em nível nacional ou por agrupamento por região ou estado. Foi possível observar que características como sexo masculino, idade

\footnotetext{
${ }^{1}$ Enfermeira. Especialista em Docência no Ensino Superior; Enfermeira. Especialista em Urgência e Emergência; Enfermeira. Docente da Faculdade Santa Maria.

2 Diretora Administrativa da Faculdade Santa Maria.

3 Enfermeira, Professora Doutora (PósDoutora), Universidade Estadual da Paraíba/UEPB. Campina Grande (PB), Enfermeira. Docente da Faculdade Santa.
} 
economicamente ativa, baixa escolaridade e heterossexualidade estiveram associados com a coinfecção TB/HIV. CONCLUSÃO: pode-se observar que a coinfecção HIV e tuberculose constitui um importante problema de saúde pública no Brasil. Assim, a proporção de coinfecção HIV/TB são fortemente influenciadas pelas características sócio demográficas da população.

Palavras-Chaves: HIV. Tuberculose. Coinfecção. Brasil.

ABSTRACT: Objective: to analyze the epidemiological aspects of tuberculosis / HIV coinfection in Brazil. Method: This is an integrative literature review, based on the following guiding question: What is the epidemiological profile of tuberculosis / HIV coinfection in the period from 2015 to 2019 in Brazil? As inclusion criteria were defined: articles published between the years2015 to 2019, in Portuguese and English, available in full and free and that addressed the theme of TB / HIV coinfection. For the exclusion criteria were used: works not available, material from congresses, class, thesis, abstract or conferences; literature reviews, as well as articles that did not address the proposed theme or were not among the defined years. The search for the articles took place through the Virtual Health Library (VHL), which indexes databases such as Medical Literature Analysis and Retrieval Sistem (MEDLINE) and Latin American and Caribbean Literature in Health Sciences (LILACS) through the terms obtained through consultation with DeCs: "HIV", "Tuberculosis", "Coinfection", "Brazil" in February 2020. Results: By analyzing the results found in this review, a pattern of prevalence of studies that presented the profile was identified sociodemographic, either at the national level or by grouping by region or state. It was possible to observe that characteristics such as male gender, economically active age, low schooling and heterosexuality were associated with TB I HIV co-infection. Conclusion: it can be seen that HIV and tuberculosis co-infection is an important public health problem in Brazil. Thus, the proportion of HIV / TB coinfection is strongly influenced by the socio-demographic characteristics of the population.

Keywords: HIV. Tuberculosis. Coinfection. Brazil. 


\section{INTRODUÇÃO}

A tuberculose (TB) configura-se mundialmente como um grave problema de saúde pública, caracterizada por ser uma doença infectocontagiosa causada pelo Mycobacterium tuberculosis, considerada uma das enfermidades com os maiores índices de mortalidade entre as doenças mais transmissíveis no mundo. Trata-se de uma patologia complexa, que envolve dimensões históricas, sócio-antropológicas e políticas (SANTOS et al., 2019).

De acordo com os dados do Ministério da Saúde, por ano, são notificados, cerca de, seis milhões de novos casos de TB em todo o mundo, que levam mais de um milhão de pessoas a óbito. No Brasil, anualmente, são notificados, aproximadamente, 70 mil casos novos e ocorrem 4,6 mil mortes devido à doença. Sua principal via de transmissão ocorre por via inalatória, através dos aerossóis, e o pulmão é o principal órgão acometido. Existem dois tipos de tuberculose: a pulmonar e a extrapulmonar, que podem infectar qualquer órgão (FONTE et al., 2019).

Segundo o Programa Nacional de Controle da Tuberculose (PNCT), existem alguns grupos populacionais que apresentam maior vulnerabilidade em adoecer, sendo que estes são considerados prioritários para o PNCT. O risco de adoecimento por TB, nessas populações, é superior à média nacional para a população em geral. No grupo de pessoas privadas de liberdade, esse risco é considerado 25 vezes maior; entre os portadores de HIV, é de 30 vezes, e chega a 67 vezes maior na população em situação de rua (NEVES et al., 2018).

No Brasil, a situação que mais confere risco de morte a pessoas que vivem com HIVIAIDS é a tuberculose ativa, e, em grande parte, o diagnóstico da coinfecção pelo HIV ocorre devido ao diagnóstico ou o tratamento da TB (WHO, 2015).

A síndrome da imunodeficiência adquirida (AIDS) já acometeu milhões de pessoas em todo o mundo. Em 2015, cerca de 36,7 milhões viviam com o vírus. No Brasil, de 1980 até junho de 2017 foram contabilizados 882.810 casos de AIDS. 
Entre 2006 e 2016, observou-se no país uma tendência de estabilização da taxa de detecção de AIDS, com uma média de 18,5/100 mil habitantes em 2016 (GRAEFF et al., 2019).

A coinfecção TB/HIV é um tema de grande relevância no país, uma vez que esses indivíduos apresentam uma alta taxa de abandono e óbito, e a TB figura como a principal doença associada ao óbito entre as pessoas vivendo com HIVIAIDS (PVHA). O estado de São Paulo se destaca por ser o estado com maior número de casos de TB e aids do Brasil e apresentar cerca de 9,9\% de casos de TB com coinfecção por HIV (CAMPOY et al., 2019).

A associação das duas doenças alterou as perspectivas de controle da TB, elevando a incidência da tuberculose e sua morbidade e mortalidade. Os indivíduos com tuberculose e com infecção pelo HIV possuem taxas de mortalidade 2,4 a 19,0 vezes maiores que os sem a coinfecção. Isto ocorre pelo desenvolvimento da multirresistência aos medicamentos, o que agrava a situação dos doentes, amplia o tempo necessário para o tratamento e, consequentemente, eleva os custos das ações de controle (MIRANDA et al., 2017).

Em 2016, o Brasil estava na lista dos 30 países com alta carga de TB/HIV, e apenas $50 \%$ dos coinfectados seguiram a recomendação da OMS iniciando há TARV oito semanas após o tratamento da TB. As taxas de mortalidade por essas doenças no país entre os anos de 2000 à 2011 foram mais elevadas em indivíduos do sexo masculino, de cor/raça negra, com idade na faixa economicamente ativa e residentes da região Sul do país. No entanto, observou uma diminuição da mortalidade, que pode estar relacionado ao uso da TARV e atualização dos protocolos utilizados no manejo clínico da coinfecção (BASTOS et al., 2019).

Assim, a sobreposição das epidemias de TB e HIV tem sido um desafio à saúde pública, principalmente, à detecção e terapêutica de ambas as condições crônicas. Dessa forma, objetivou-se analisar, a partir de uma revisão integrativa da literatura, qual o perfil epidemiológico da coinfecção tuberculose/HIV no período de 2015 a 2019 no Brasil. 


\section{MÉTODO}

Tratou-se de um estudo de revisão integrativa com etapas pré-determinadas, de caráter exploratório com abordagem quantitativa. Para Mendes et al. (2008) a revisão integrativa consiste na construção de uma análise ampla da literatura, contribuindo para discussões sobre métodos e resultados de pesquisas, a partir de determinado objetivo. Dessa forma, a construção da revisão percorrer seis etapas distintas, são elas: Estabelecimento da hipótese ou questão da pesquisa; a busca na literatura; categorização dos estudos; avaliação dos estudos incluídos na revisão; interpretação dos resultados e, por fim, apresentação da revisão.

Para construção da revisão definimos o tema e a questão norteadora: Qual o perfil epidemiológico da coinfecção tuberculose/HIV no período de 2015 a 2019 no Brasil? Como critérios de inclusão definiram-se: artigos publicados entre os anos de 2015 e 2019, em português e inglês, disponíveis na integra e gratuitos e que abordassem a temática de coinfecçao TB/HIV. Para os critérios de exclusão foram utilizados: trabalhos não disponíveis, material de congressos, aula, tese, resumo ou conferencias; revisões da literatura, bem como artigos que abordavam o tema proposto. A busca pelos artigos ocorreu pela Biblioteca Virtual de Saúde (BVS), que indexa bases de dados como Medical Literature Analysis and Retrieval Sistem (MEDLINE) e Literatura Latino-Americana e do Caribe em Ciências da Saúde (LILACS) através dos termos obtidos por meio de consulta aos DeCs: "HIV", "Tuberculose", "Coinfecção", "Brasil".

A busca foi realizada no mês de fevereiro de 2020 que, inicialmente, obteve um quantitativo de 73 artigos. Onze artigos estavam duplicados nas diferentes bases de dados, restando um total de 62 artigos que tiveram seus títulos, e posteriormente resumos, lidos. Após a leitura de títulos e resumos, 29 artigos foram excluídos por não se adequarem ao tema proposto. Restaram então 33 artigos, dos quais foram retirados 24 devido aos critérios de inclusão e exclusão. Por fim, restaram somente 9 artigos que, após a leitura completa, foram incluídos nesta revisão integrativa. 
Assim, a leitura criteriosa desses artigos possibilitou extrair dos mesmos informações importantes para a construção desta revisão integrativa e ainda o confronto com outros autores enriqueceu o debate proposto dentro da temática abordada.

Figura 1: Fluxograma Metodológico. Fonte: Dados da Pesquisa, 2020.

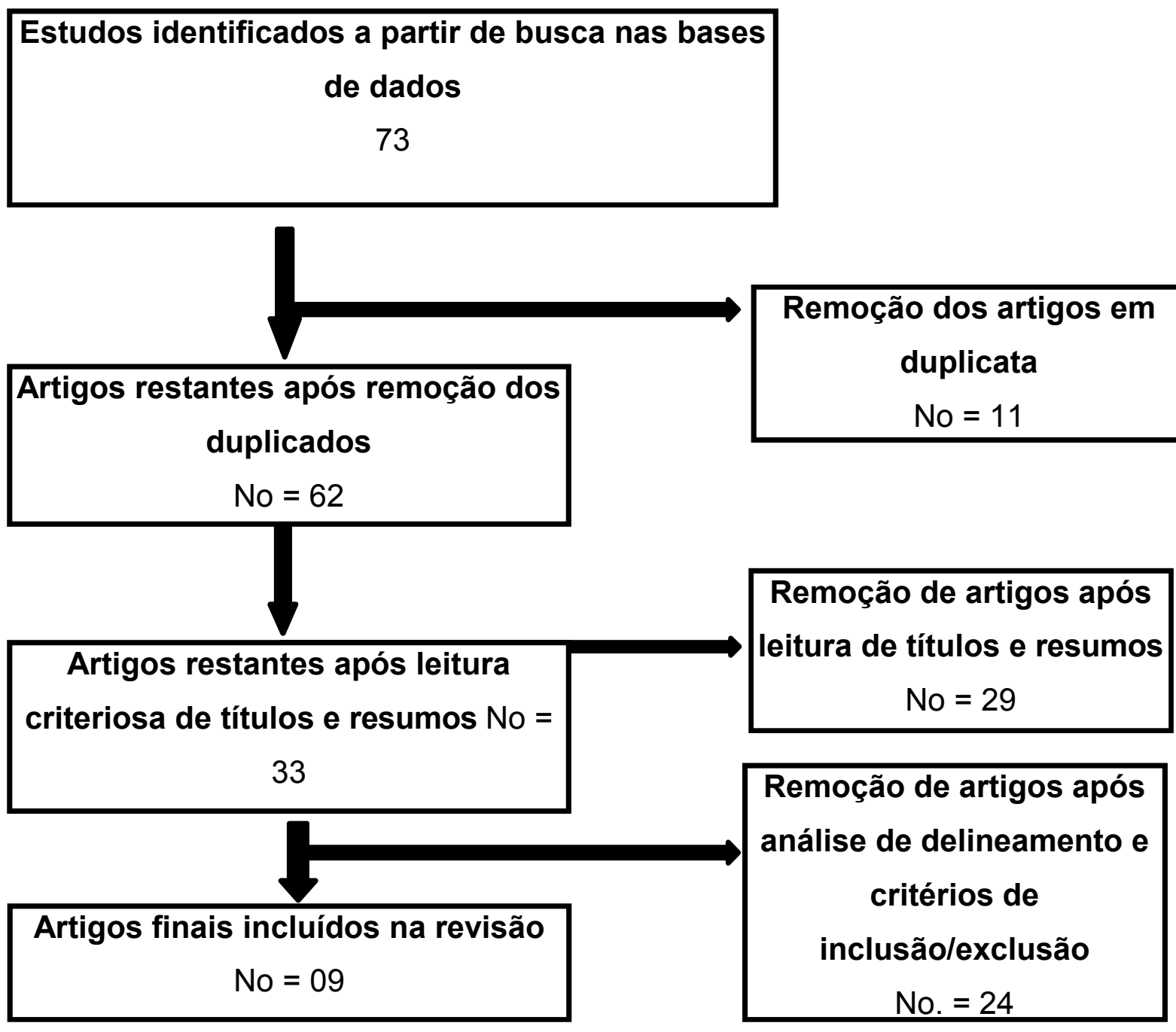

\section{RESULTADOS}

Tomando como base os autores selecionados, o quadro1 categoriza os estudos de acordo com o título, revista de publicação, ano e local tipo de pesquisa. 
A categorização dos estudos é importante, pois permite que cada documento analisado possa ser minimamente apresentado de acordo com suas características.

Quadro 1: Categorização dos estudos quanto ao autor, revista, loca e tipo de estudo.

\begin{tabular}{|c|c|c|c|c|}
\hline TITULO & AUTOR/ANO & REVISTA & LOCAL & $\begin{array}{l}\text { TIPO DE } \\
\text { ESTUDO }\end{array}$ \\
\hline $\begin{array}{l}\text { Teste de HIV, terapia antirretroviral e } \\
\text { desfechos do tratamento em novos } \\
\text { casos de tuberculose no Brasil, } 2011\end{array}$ & $\begin{array}{l}\text { Torrens et al. } \\
\quad(2016)\end{array}$ & $\begin{array}{c}\text { Revista } \\
\text { Panamericana de } \\
\text { Saúde Pública }\end{array}$ & Brasil & Transversal \\
\hline $\begin{array}{l}\text { Fatores associados à coinfecção } \\
\text { tuberculose e HIV: o que apontam os } \\
\text { dados de notificação do Estado do } \\
\text { Amazonas, Brasil, 2001-2012 }\end{array}$ & $\begin{array}{l}\text { Magno et al., } \\
\quad(2017)\end{array}$ & $\begin{array}{l}\text { Caderno Saúde } \\
\text { Pública }\end{array}$ & Brasil & Quantitativo \\
\hline $\begin{array}{l}\text { Análise temporal dos casos notificados } \\
\text { de tuberculose e de coinfecção } \\
\text { tuberculose-HIV na população } \\
\text { brasileira no período entre } 2002 \text { e } 2012\end{array}$ & Gaspar et al., & $\begin{array}{l}\text { Jornal Brasileiro } \\
\text { de Pneumologia }\end{array}$ & Maranhão & Observacional \\
\hline $\begin{array}{c}\text { Características clínico-epidemiológicas } \\
\text { da coinfecção por tuberculose e HIV e } \\
\text { sua relação com o İ́ndice de } \\
\text { Desenvolvimento Humano no estado } \\
\text { do Mato Grosso do Sul, Brasil }\end{array}$ & $\begin{array}{l}\text { Baldan et al., } \\
\quad(2017)\end{array}$ & $\begin{array}{l}\text { Revista Pan- } \\
\text { Americana de } \\
\text { Saúde }\end{array}$ & $\begin{array}{l}\text { Mato } \\
\text { Grosso do } \\
\text { Sul }\end{array}$ & Descritivo \\
\hline $\begin{array}{l}\text { Os significados da comorbidade para } \\
209 \text { os pacientes vivendo com TB/HIV: } \\
\text { repercussões no tratamento }\end{array}$ & $\begin{array}{l}\text { Silva et al., } \\
\quad(2015)\end{array}$ & $\begin{array}{c}\text { Revista de Saúde } \\
\text { Coletiva }\end{array}$ & $\begin{array}{l}\text { Rio de } \\
\text { aneiro }\end{array}$ & Qualitativa \\
\hline $\begin{array}{l}\text { Infecção latente por tuberculose entre } \\
\text { pessoas com HIVIAIDS, fatores } \\
\text { associados e progressão para doença } \\
\text { ativa em município no Sul do Brasil }\end{array}$ & $\begin{array}{l}\text { Santos et al., } \\
\quad(2017)\end{array}$ & $\begin{array}{c}\text { Caderno de Saúde } \\
\text { Pública }\end{array}$ & Paraná & Descritivo \\
\hline $\begin{array}{c}\text { Controle da Tuberculose em pessoas } \\
\text { vivendo com HIV/aids }\end{array}$ & $\begin{array}{c}\text { Magnabosco et } \\
\text { al., (2016) }\end{array}$ & $\begin{array}{l}\text { Revista Latino- } \\
\text { Americana de } \\
\text { Enfermagem }\end{array}$ & São Paulo & Quantitativo \\
\hline $\begin{array}{l}\text { Mortality related to tuberculosis- } \\
\text { HIVIAIDS co-infection in Brazil, 2000- } \\
\text { 2011: epidemiological patterns and } \\
\text { time trends }\end{array}$ & $\begin{array}{l}\text { Lima et al., } \\
\quad(2016)\end{array}$ & $\begin{array}{c}\text { Caderno de Saúde } \\
\text { Pública }\end{array}$ & Fortaleza & Descritivo \\
\hline $\begin{array}{l}\text { Fatores associados à coinfecção } \\
\text { tuberculose/HIV no período 2001-2011 }\end{array}$ & $\begin{array}{l}\text { Pereira et al., } \\
\quad(2018)\end{array}$ & $\begin{array}{l}\text { Revista Online de } \\
\text { Pesquisa }\end{array}$ & $\begin{array}{l}\text { Rio de } \\
\text { Janeiro }\end{array}$ & Transversal \\
\hline
\end{tabular}


quadro 2 mostra os resultados encontrados pelos autores selecionados e os objetivos dos respectivos estudos desenvolvidos.

\begin{tabular}{|c|c|c|c|}
\hline AUTO & R/ANO & OBJETIVO & RESULTADO \\
\hline $\begin{array}{l}\text { Torrens } \\
\text { (2016) }\end{array}$ & et & $\begin{array}{l}\text { Avaliar a implementação de } \\
\text { intervenções relacionadas } \\
\text { ao HIV para pacientes com } \\
\text { al. tuberculose (TB) assim } \\
\text { como os desfechos do th } \\
\text { tratamento de TB nos } \\
\text { pacientes coinfectados com } \\
\text { HIV no Brasil em 2011. }\end{array}$ & $\begin{array}{l}\text { O linkage entre os bancos de dados nacionais de } \\
\text { tuberculose e HIV proporcionou um parâmetro de } \\
\text { comparação conveniente para o monitoramento } \\
\text { continuo do teste de HIV, uso de TAR e desfechos do } \\
\text { tratamento de TB em pacientes coinfectados com TB- } \\
\text { HIV. A associação entre TAR e sucesso do tratamento } \\
\text { de TB é mais uma evidência que respalda a iniciação } \\
\text { oportuna da TAR em todos os pacientes coinfectados } \\
\text { com TB-HIV. }\end{array}$ \\
\hline $\begin{array}{l}\text { Magno } \\
(2017)\end{array}$ & et & $\begin{array}{l}\text { Estimar a prevalência daE } \\
\text { coinfecção TB/HIV, avaliar ad } \\
\text { notificação dos dois agravosa } \\
\text { de maneira temporal, por } \\
\text { meio de relacionamento de } \\
\text { bases de dados, e buscar a } \\
\text { fatores associados às } \\
\text { notificações de AIDS e TB. }\end{array}$ & $\begin{array}{l}\text { A prevalência estimada de coinfecção TB/HIVIAIDS no } \\
\text { Estado do Amazonas foi de } 7,7 \% \text { pelo relacionamento } \\
\text { das bases de dados do SINAN AIDS e TB, enfatizando } \\
\text { a importância do HIV como fator isolado para a } \\
\text { ocorrência da TB nesta população. Do ponto de vista } \\
\text { clínico, o grupo de pacientes em que a TB ocorreu } \\
\text { após terem sido identificados como PVHIV foi o mais } \\
\text { representativo, mesmo existindo recomendações para } \\
\text { a redução da TB nesta população, o que colaboraria } \\
\text { para a diminuição da mortalidade pela coinfecção. }\end{array}$ \\
\hline $\begin{array}{l}\text { Gaspar } \\
(2016)\end{array}$ & et & $\begin{array}{l}\text { Investigar os casosii } \\
\text { notificados de tuberculose ev } \\
\text { l. } \text { de sua coinfecção com od } \\
\text { HIV na população brasileirala } \\
\text { no período entre } 2002 \text { e } \\
2012 \text {. }\end{array}$ & $\begin{array}{l}\text { O presente estudo evidencia a tuberculose como um } \\
\text { vimportante problema de saúde pública no Brasil, uma } \\
\text { vez que as metas estabelecidas de cura e controle da } \\
\text { doença ainda não foram alcançadas. Ademais, o } \\
\text { aumento vertiginoso na incidência de tuberculose-HIV } \\
\text { em mulheres, idosos e nas regiões Norte e Nordeste } \\
\text { evidencia a feminização, a transição etária e a } \\
\text { pauperização pelo HIV. }\end{array}$ \\
\hline $\begin{array}{l}\text { Baldan } \\
(2017)\end{array}$ & et & $\begin{array}{l}\text { Verificar os aspectos } \\
\text { epidemiológicos da } \\
\text { coinfecção por tuberculose } \\
\text { (TB) e HIV, no estado do } \\
\text { Mato Grosso do Sul, Brasil, } \\
\text { e sua associação com o } \\
\text { índice de Desenvolvimento } \\
\text { Humano. }\end{array}$ & $\begin{array}{l}\text { Observou-se a associação entre casos de coinfecção } \\
\text { por TB/HIV e IDH em áreas com maior densidade } \\
\text { populacional, indicando a necessidade de adoção de } \\
\text { estratégias específicas de acordo com as } \\
\text { características de cada localidade. }\end{array}$ \\
\hline $\begin{array}{l}\text { Silva } \\
(2015)\end{array}$ & et & 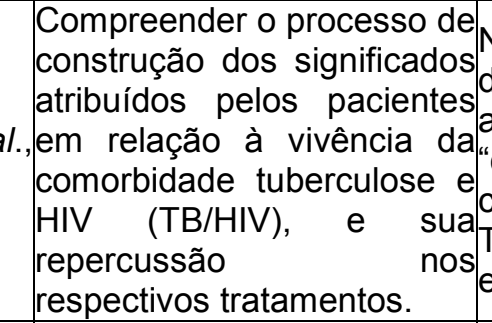 & $\begin{array}{l}\text { Na perspectiva do paciente, vivenciar a dupla condição } \\
\text { de doença é uma experiência marcante, limitante, } \\
\text { assustadora e considerada por alguns como um } \\
\text { "castigo". Podemos afirmar que diversos fatores } \\
\text { compõem os significados da vivência da comorbidade } \\
\text { TB/HIV para os pacientes. Tais fatores encontram-se } \\
\text { entrelaçados numa trama significativa. }\end{array}$ \\
\hline $\begin{array}{l}\text { Santos } \\
(2017)\end{array}$ & et & 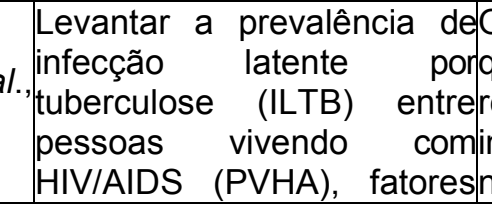 & $\begin{array}{l}\text { O estudo contribuiu para o avanço do conhecimento no } \\
\text { que tange à prevalência da ILTB em um serviço } \\
\text { referência para PVHA, encontrando associação à } \\
\text { infecção, ao sexo masculino, à condição prisional e ao } \\
\text { número mais elevado de linfócitos T. Alguns casos }\end{array}$ \\
\hline
\end{tabular}




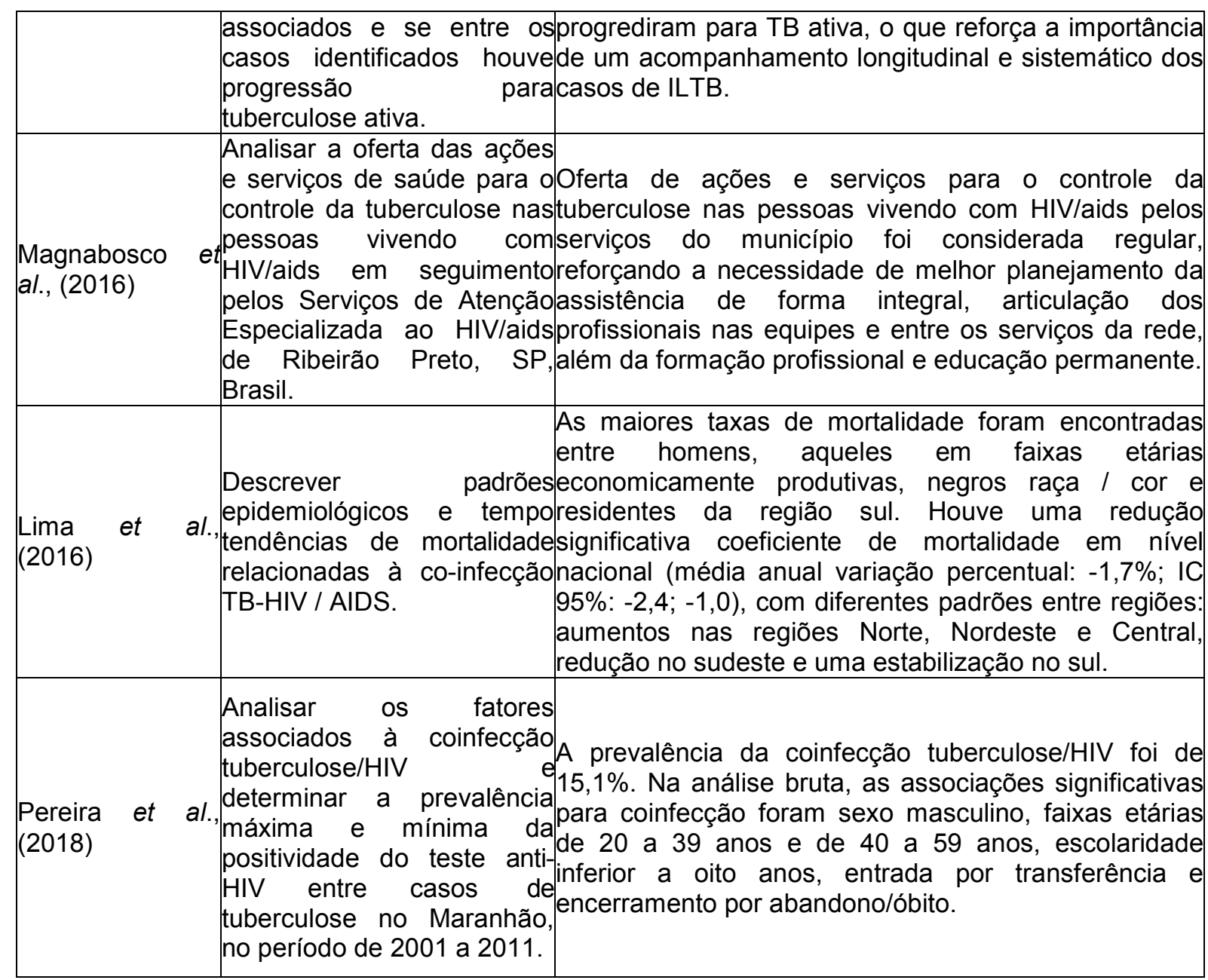

\section{DISCUSSÃO}

Pela análise dos resultados encontrados nesta revisão, identificou-se um padrão de prevalência de estudos que apresentaram o perfil sociodemográfico, seja em nível nacional ou por agrupamento por região ou estado. Foi possível observar que características como sexo masculino, idade economicamente ativa, baixa escolaridade e heterossexualidade estiveram associados com a coinfecção TB/HIV.

O estudo realizado por Pereira et al. (2018) buscou analisar os fatores associados à coinfecção tuberculose/HIV e determinar a prevalência máxima e mínima da positividade do teste anti-HIV entre casos de tuberculose. Os autores puderam concluir que existe uma alta prevalência de testes anti-HIV positivos entre 
portadores de TB, principalmente, entre o sexo masculino com faixas etárias de 20 a 39 anos e de 40 a 59 anos, com escolaridades inferiores há oito anos, dados que reafirmam os achados da pesquisa.

Em concordância com Pereira et al. (2018), Lima et al. (2016) em suas pesquisas desenvolvidas no Brasil sobre padrões epidemiológicos e tendências temporais da mortalidade relacionada à coinfecção TB-HIVIAIDS, mostrou que os maiores coeficientes de mortalidade foram verificados no sexo masculino, grupos etários economicamente produtivos, raça/cor negra e residentes da Região Sul.

Frente a estes resultados, Gaspar et al., (2016) ao observar a incidência de tuberculose por sexo, percebe-se que essa é duas vezes maior no sexo masculino do que no feminino. Esse dado substancia a indicação de que, no Brasil, a tuberculose atinge prioritariamente o sexo masculino, tendo esses duas vezes mais chance de adoecer. É necessário comentar que houve uma redução mais acentuada na incidência de tuberculose no sexo feminino, provavelmente pelo maior cuidado com a saúde por parte das mulheres. Entretanto, o sexo feminino desponta na incidência da coinfecção tuberculose-HIV, indicando um maior número de novas mulheres soropositivas.

Santos et al. (2017) ao analisar a prevalência de infecção latente por tuberculose (ILTB) entre pessoas vivendo com HIVIAIDS (PVHA), observou que ser do sexo masculino, estar em situação de privação de liberdade e contagem de linfócito TCD4+ mais elevada foram associados à ILTB.

De acordo com Baldan et al., (2017) os fatores que interferem na maior ocorrência de casos em homens podem estar relacionados a diversas condições, seja de ordem biológica, cultural e organizacional, devido à inflexibilidade nos horários de atendimento, o que dificulta o acesso dos trabalhadores, ou ainda a possibilidade de subdiagnóstico em mulheres.

Este estudo demonstrou que há um maior número de casos de coinfecção TB/HIV em adultos jovens, refletindo uma maior proporção de TB nesse grupo ou realização de testes de HIV mais frequente no referido grupo. O estilo de vida da população nessa faixa etária, com comportamentos vulneráveis, pode resultar em maior exposição ao HIV e ao M. tuberculosis (PEREIRA et al., 2018). 
O nível educacional representa um determinante social da saúde, uma vez que o acesso à educação influencia na capacidade do sujeito assimilar as informações de promoção de saúde, de prevenção de doenças e na adesão ao tratamento. Dessa forma, na presente pesquisa, observou-se uma baixa escolaridade da população estudada na maioria dos estudos analisados.

Sabe-se que, a TB e o vírus da imunodeficiência humana apresentam desafios enormes para o bem-estar social, econômico, de saúde e de desenvolvimento das populações em todo o mundo (TORRENS et al., 2016). O fenômeno da comorbidade TB/HIV agrava o panorama da tuberculose, uma das mais antigas epidemias da humanidade. Ambas afetam populações menos favorecidas, vulneráveis socialmente e nas faixas etárias mais produtivas da vida, já que se trata de duas doenças transmissíveis, consideradas crônicas e com tratamentos distintos, porém semelhantes em diversos sentidos (SILVA et al., 2015).

Silva et al. (2015) afirma que a comorbidade TB/HIV acirra a convivência dos pacientes com as duas doenças e requer terapêutica adicional, que traz consigo novos efeitos colaterais e interações medicamentosas, demandando práticas e estratégias na assistência específicas para o acompanhamento e adesão ao tratamento. Trata-se de duas enfermidades que a priori se aproximam pelo caráter de cronicidade e, portanto, da necessidade de cuidados específicos e de longo tempo.

Para Baldan et al., (2017) o impacto negativo da inter-relação entre a TB e o HIV representa um desafio para o alcance das metas estabelecidas pela OMS, pois o diagnóstico da doença nos indivíduos HIV positivo costuma ser tardio, em decorrência de falhas de comunicação entre os serviços de referência para o atendimento às PVHA e com TB.

O Ministério da Saúde recomenda que todos os pacientes com tuberculose sejam submetidos à testagem sorológica para o HIV. Esta medida possibilita um diagnóstico precoce da infecção pelo HIV, permitindo, quando indicado, o início da terapia antirretroviral, gerando uma consequente diminuição de sua morbidade e mortalidade e constituindo-se em uma importante ferramenta para a elaboração e execução de políticas públicas (MIRANDA et al., 2017). 
Contudo, Barbosa et al., (2014) afirma que a maioria dos casos de TB e de HIV ocorre em regiões com menores recursos econômicos, dificultando o diagnóstico, o tratamento e o controle da infecção. Além disso, apesar das grandes conquistas no tratamento tanto da TB como do HIVIAIDS, os serviços de saúde pública nem sempre conseguem atender às demandas para o controle das epidemias.

Segundo Miranda et al., (2017), o tratamento de pacientes coinfectados com TB-HIV é difícil, requer tempo e uma variedade de medicamentos. A adesão por parte dos pacientes é difícil devido à carga extra da tomada de drogas, resultando em maior taxa de inadimplência. Devido a esta dificuldade e à fraca adesão ao tratamento da tuberculose, é importante a construção de novas estratégias para o monitoramento do tratamento, levando em consideração as características da coinfecção conforme recomendado pelo Plano Nacional de Controle da Tuberculose.

Em relação à oferta desses serviços de saúde para o controle da TB nas PVHA, Magnabosco et al., (2016) reforça a necessidade de melhor planejamento da assistência a cada indivíduo. Orientações sobre as doenças pautadas na abordagem individual configuram importantes elementos para educação e empoderamento da pessoa para a percepção de sinais e sintomas da doença, da mesma maneira que para melhorar a adesão ao tratamento e fortalecimento do vínculo entre o profissional e indivíduo.

\section{CONCLUSÃO}

Nessa revisão integrativa pode-se observar que a coinfecção HIV e tuberculose constitui um importante problema de saúde pública no Brasil. Por meio dos artigos analisados pode-se concluir que grande número de HIV positivos desenvolve tuberculose, e a coinfecão de TB/.HIV está associada a adultos jovens, do sexo masculino e com baixa escolaridade o que os autores associam ao abandono do tratamento, refletindo na qualidade de vida e, posteriormente, a morte. 
Assim, a proporção de coinfecção HIV/TB são fortemente influenciadas pelas características sócio demográficas da população.

\section{REFERÊNCIAS BIBLIOGRÁFICAS}

BALDAN, S. S., et al. Características clínico-epidemiológicas da coinfecção por tuberculose e HIV e sua relação com o Índice de Desenvolvimento Humano. Revista Pan-Amazonica de Saúde. v. 8, n. 3, p. 59-67. 2017.

BASTOS, S. H. et al. Perfil Sociodemográfico e de saúde da coinfecção tuberculose/HIV no Brasil: revisão sistemática. Revista Brasileira de Enfermagem. Brasília, v.72, n.5 p. 16, 2019. https://doi.org/10.1590/0034-7167-2018-0285.

CAMPO, L. T. et al. Qualidade e gestão da atenção à coinfecção tuberculose e hiv no estado de São Paulo. Texto e contexto - Enfermagem. Florianópolis. v.28, n. 29, Jul. 2019. https://doi.org/10.1590/1980-265x-tce-2018-0166.

FONTES, G. J. F. et al. Perfil epidemiológico da tuberculose no Brasil no período de 2012 a 2016. Revista Brasileira de Educação em Saúde, v. 9, n. 1, p. 19-26, jan-mar, 2019.

GASPAR, R. S., NUNES, N., NUNES, M., RODRIGUES, V. P. Análise temporal dos casos notificados de tuberculose e de coinfecção tuberculose-HIV na população brasileira no período entre 2002 e 2012. Jornal Brasileiro Pneumologia. v.42, n. 6, p.416-422, 2016. http://dx.doi.org/10.1590/S1806-37562016000000054

GRAEFF, S. V. B. et al. Aspectos epidemiológicos da infecção pelo HIV e da aids entre povos indígenas. Revista de Saúde Pública. v. 53, n. 9, Set, 2019.

LIMA, M. S., et al. Mortality related to tuberculosis-HIVIAIDS co-infection in Brazil, 2000- 2011: epidemiological patterns and time trends. Caderno de Saúde Pública, Rio de Janeiro, v. 32, n. 10, out, 2016.

MAGNABOSCO, G. T., LOPES, L. M., ANDRADE, R. L. P., BRUNELLO, M. E. F., MONROE, A. A., VILLA, T. C. S. Tuberculosis control in people living with HIVIAIDS. Revista LatinoAmericana de Enfermagem. v. 24, 2016. DOI: http://dx.doi.org/10.1590/1518-8345.1187.2798.

MAGNO, E. S et al. Fatores associados à coinfecção tuberculose e HIV: o que apontam os dados de notificação do Estado do Amazonas, Brasil, 2001-2012. Caderno de Saúde Pública. v.33, n. 5, 2017.

MIRANDA, L. O., et al. Aspectos epidemiológicos da coinfecção Tuberculose/HIV no Brasil: revisão integrativa. Revista Prevenção de Infecção e Saúde. v.3, n. 3, p. 59-70, 2017. Available from: http://www.ojs.ufpi.br/index.php/nupcis/article/view/5848.

NEVES, D. C. O. et al. Aspectos epidemiológicos da tuberculose nas Regiões de Integração do estado do Pará, Brasil, no período entre 2005 e 2014. Revista Pan-Amazonica Saúde v.9 n.3, set. 2018. http://dx.doi.org/10.5123/s2176-62232018000300003.

PEREIRA, L. F. B.; SOARES, D. L.; SILVA, T. C. et al. Fatores associados à coinfecção tuberculose/HIV no período 2001-2011. Revista Online de Pesquisa. v. 10, n. 4, p. 10261031, out/dez, 2018. 
SANTOS, D. T., et al. Infecção latente por tuberculose entre pessoas com HIVIAIDS, fatores associados e progressão para doença ativa em município no Sul do Brasil. Caderno Saúde Pública. v. 33, n. 8, 2017.

SANTOS, J. G. C. et al., Perfil Clínico e Epidemiológico da Tuberculose em Alagoas de 2008 a 2017. Revista Saúde e Desenvolvimento. v.13, n.14, 2019.

SILVA, J. B., et al. Os significados da comorbidade para os pacientes vivendo com TB/HIV: repercussões no tratamento. Revista de Saúde Coletiva, Rio de Janeiro, v.25, n. 1, p. 209229, 2015.

TORRENS, A., BARTHOLOMAY, P., SILVA, S., KHOGALI, M., VERDONCK, K., BISSELL, K. HIV testing, antiretroviral therapy, and treatment outcomes in new cases of tuberculosis in Brazil, 2011. Revista Pan-Anamericana de Saúde Publica. v.39, n. 1, p. 26- 31, 2016. 